lampe nach Rompe und Thouret ${ }^{5}$ mit einer Flächenhelligkeit von 200,000 Stilb verwandt. Abb. 3 * gibt eine Aufnahme von Aerosolteilchen wieder, wie sie mit diesem Gerät erhalten wurde. Die Gesamtvergrößerung der Aufnahmen ist etwa 125-fach. Als Aufnahmematerial diente der Agfa-Ultra-Isopan-Film mit einer Empfindlichkeit von 23/10 Din ${ }^{\circ}$, Kleinbildformat. Die Belichtungszeiten richteten sich nach den jeweiligen Aerosolen. Bei feinsten Nebeln, z. B. Chlorsulfonsäure-Nebel, kurz nach der Herstellung, ist eine Belichtungszeit von $10-15 \mathrm{sec}$ notwendig. Die Teilchen zeigen in dieser Zeit fast keine Fallbewegung.

5 R. R o m p e u. W. Thouret, Z. techn. Physik 17, 377 [1936].

* s. Tafel S. 144 b.
In Abb. 4 sind einige Verteilungskurven der Teilch€ngrößen wiedergegeben, welche an ein und demselben Nebel zu verschiedenen Zeiten erhalten wurden. Sie zeigen deutlich die zeitliche Verschiebung der Teilchenradien zu größeren Werten mit zunehmendem Alter des Aerosols, besonders schön beim Chlorsulfonsäure-Nebel, dagegen zeigt sich der Zinkchlorid-Nebel als recht gleichmäßig auch über längere Zeiten hinweg.

Die Ermittlung der Radien erfolgt durch Projektion des Negatives bei bekannter Gesamtvergrößerung auf Millimeterpapier. Da die Fallzeit bekannt ist, kann auf die Fallzeit 1 sec umgerechnet werden; die Radien sind aus einmal ausgerechneten Tabellen sofort zu entnehmen.

\title{
Über ein Modell der für Ausleuchtung sensibilisierten Phosphore
}

\author{
Von Peter Brauer \\ Aus der Osram G.m.b.H., Zweigstelle Mosbach (Baden) \\ (Z. Naturforschg. 5 a, 156-158 [1950]; eingegangen am 19. Dezember 1949)
}

\begin{abstract}
Zum Verständnis der zwei Aktivatoren enthaltenden, für Ausleuchtung sensibilisierten Phosphore wird ein Modell bzw. Termschema vorgeschlagen und mit der Erfahrung verglichen. Wesentlich ist dabei, daß der eine Aktivator die Bildung besetzter, der andere die Bildung unbesetzter Aktivatorterme veranlaßt.
\end{abstract}

$\mathrm{D}$ ie vom Verf. ${ }^{1}$ beschriebenen, von $\mathrm{R}$. T o m a s che k, dem Verf. und O. Deuts chbein ${ }^{3,4,5,6}$ im Jahre 1941 aufgefundenen Erdalkalisulfidphosphore mit zwei Aktivatoren ${ }^{7}$ haben außer ihrer stark erhöhten Empfindlichkeit für ausleuchtendes, meist ultrarotes, Licht noch eine Anzahl früher unbekannter, nicht minder bemerkenswerter Eigenschaften. Diese brachten es mit sich, daß die Phosphore selbst qualitativ nicht mit Hilfe der bekannten Modelle verstanden werden konnten. Das war um so merkwürdiger, als bei Weglassung eines der beiden Aktivatoren sich ein Phosphor mit normalem Verhalten ergab ${ }^{1}$. Da es sich bei den Mischphosphoren um eine Art Zweibandenphosphore handelt, wurde versucht ${ }^{2}$, die bei der Gruppe der Zink-Cadmiumsulfide so erfolgreiche Theorie der Zweibandenphosphore von M. Schön ${ }^{8}$, erweitert durch einen Gedanken von

1 P. B r a u e r, Z. Naturforschg. 1, 70 [1946].

2 P. B r a u e r, Z. Naturforschg. 2a, 238 [1947].

3 R. Tom a s chek u. P. B rauer, Forsch. - Ber. Zentrale wiss. Berichtswes. (ZWB) 1695/1.

4 R. Tom a s chek, P. B rauer u. J. Häckel, ebenda 1695/2.

5 P. B r a u e r, ebenda $1695 / 3$.
W. Schottky ${ }^{9}$, anzuwenden. Es gelang so zwar, die Verschiedenheit der Emissionsspektren beim Ausheizen und Ausleuchten zu verstehen, an anderen Stellen jedoch versagte sie. Insbesondere gelang es nicht, die aus dem Modell folgende Kinetik mit der Erfahrung in Übereinstimmung zu bringen.

Verf. möchte im folgenden ein Modell vorschlagen, welches, soweit bis jetzt erkennbar, die bestehenden Schwierigkeiten beseitigt. Einen für das Modell wesentlichen Gedanken, nämlich besetzte mit unbesetzten Aktivatortermen zu kombinieren, um so-

6 P. B r a u e r, ebenda $1695 / 4$.

7 Die in Frage kommende Gruppe von Phosphoren wurde - offenbar unabhängig von uns - in der Firma $\mathrm{S}$ i e m ens u. Hals ke aufgefunden. Sie wurde außerdem später beschrieben von F. U rbach, D. Pearlman u. W. He m m ending er, J. opt. Soc. America 36, 374 [1946]; R. T. Ellickson, ebenda 36, 264 [1946]; W. W. Antonow-R o m a now s ki, Ber. Akad. Wiss. UdSSR 54, 791 [1946] (nach Chem. Zbl. 1947, 971) u. a. 8 M. S c hö n, Z. Physik 119, 463 [1939], Forsch. u. Fortschr. 19, Nr. 23/24 [1943], Ann. Physik (6) 3, 333 [1948].

9 den der Verf. einer freundlichen, mündlichen Mitteilung Hrn. Prof. Dr. W. S ch ot t ky s verdankt (siehe hierzu die unter 2 angegebene Arbeit). 
wohl den Wechsel des Emissionsspektrums als die Speich€reigenschaften zu erklären, hat, wie der Verf. zwar erst später fand, aber hier betonen möchte, schon vorher $\mathrm{F}$. A. K r ö g e r veröffentlicht ${ }^{10}$. In Abb. la ist das vereinfachte Termschema eines SrS-Eu-Phosphors auf Grund seiner Phänomenologie ${ }^{11}$ dargestellt, wie es aussehen könnte, wenn man die Schönsche Theorie der (einfachen) Kristallphosphore ${ }^{12}$ auf diesen Fall überträgt. In Abb. $1 \mathrm{~b}$ ist entsprechend das Termschema eines SrS-Sm-Phosphors aufgetragen. Hierbei wurde aber angenommen, daß der Aktivatorterm ein von Elektronen unbesetzter Term ist. Denn es ist, hierauf hat W. S chottky ${ }^{13}$ hingewiesen, in Anbetracht der positiven Überschußladung des, wie das Emissionsspektrum zeigt, dreiwertigen $\mathrm{Sm}^{3+}$-Ions gegenüber den zweiwertigen Gitterbausteinen des Grundgitters, wahrscheinlicher, daß als Folge der Anregung des $\mathrm{Sm}^{3+}$ ein Defektelektron ins Gitter läuft. Wir machen also hier Gebrauch von der formalen Möglichkeit, in der Theorie der Kristallphosphore alle Ladungsvorzeichen umzukehren. Entsprechend sind Haftstellen für Defektelektronen eingezeichnet. Abb. $1 \mathrm{a}$ und $1 \mathrm{~b}$ stellen also zwei einzelne, normal funktionierende Phosphore dar. Der SrS-Eu-Sm-Mischphosphor muß jetzt durch die ganze Abb. 1 unter Wegdenken der gestrichelten senkrechten Trennungslinie beschrieben werden. Dies ist tatsächlich der Fall. - Erregt man z. B. in der SrS-Eu-Erregungsbande (Pfeil 1, Abb. 1a) ${ }^{1.5}$, so werden die Elektronen nicht allein in den dem SrS-Eu eigentümlichen Haftstellen, sondern vor allem in den zahlreichen tiefen $\mathrm{Sm}^{3+}$-Termen, die ja unbesetzt sind, gespeichert, wodurch sich die Verlangsamung des Abklingens ${ }^{3}$ beim Mischphosphor und die vergrößerte ausleuchtbare Lichtsumme ${ }^{1.3}$ erklärt. Folgerichtig erhielten wir bei Präparaten mit Eu-Überschuß beim allmählichen Ausheizen zwei Maxima der Helligkeits-TemperaturKurve, von denen das den seichteren Haftstellen des SrS-Eu (Pf. 3) entsprechende bei zunehmendem SmGehalt relativ gegenüber dem neuen, durch das sensibilisicrende Sm hervorgerufene, tieferen Haftstellen (Pf. 6 u. 7) entsprechende Maximum zurücktrat. Erregt man gleichzeitig im Sm auch (Pf.4), so vermindern sich durch Ablösung von Defektelektronen von den $\mathrm{Sm}^{3+}$-Termen, die Haftmöglichkeiten für die Elektronen: die ausleuchtbare Eu-Lichtsumme vermindert sich $^{3}$. - Sensibilisierte Ausleuchtung erfolgt

10 F. A. K r ög e r, Some Aspects of the Luminsescence of Solids, Amsterdam 1948, S. 40.

11 s. Fußnote 1-6; zur Einzeichnung der thermisch zu überwindenden Stufe von $\sim 0,5 \mathrm{eV}$ s. Fußnote 18 in 1 . durch Wiederbefreiung der Elektronen infolge der starken UR-Eigenabsorption des $\mathrm{Sm}$ von $1,2 \mathrm{eV}$ des $\mathrm{Sm}^{3+}$ (Pf. 6) ${ }^{1,2.4}$, wobei, wie auch bei der Erregung, das thermische Verhalten das Vorhandensein einer thermisch zu überwindenden Stufe anzeigt (Pf. 7 bzw. 2) ${ }^{2.6}$. Im voll erregten Phosphor gelangt ein gewisser Teil zur Rekombination bei Eu-Störstellen und gibt Anlaß zum Leuchten mit $\mathrm{Eu}^{2+}$-Spektrum. (Der Rest speist Verlustprozesse ${ }^{4}$.) Im teilweise ausgeheizten bzw. abgeklungenen Phosphor hingegen dient ein Teil zur Auffüllung der durch Ausheizen

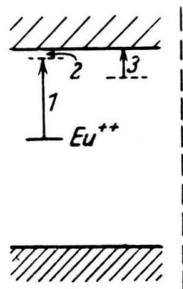

1a)

2)

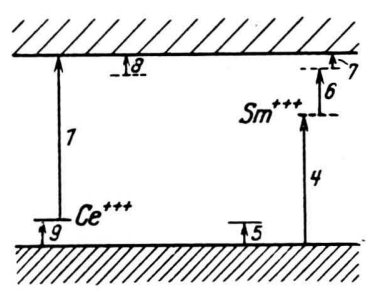

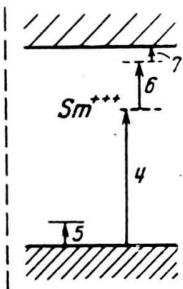

1b)
Abb. 1. Vereinfachtes Termschema von a: SrS-Eu, b: SrS-Sm, a und b zusammen mit weggelassener Trennungslinie: SrS-Eu-Sm.

Abb. 2. Vereinfachtes Termschema von SrS-Ce-Sm.

teilweise geleerten gewöhnlichen Haftstellen; der zum Leuchten gelangende Teil ist kleiner. Da beim Ausheizen die im sensibilisierten Phosphor (also am $\mathrm{Sm}^{3+}$ ) in sehr tiefen Haftstellen gespeicherten Elektronen infolge der notwendig hohen Ausheiztemperatur (etwa $650^{\circ} \mathrm{K}$ ) offenbar vorwiegend Verlustprozesse speisen ${ }^{1,14}$, muß sich der Gütefaktor $u$ der Ausleuchtung (es ist $u=d L_{A} / d L_{H a}$, wo $L_{A}$ die ausgeleuchtete Lichtsumme und $L_{H u}$ ihr ausheizbares Äquivalent ist) kleiner ergeben als bei gefüllten gewöhnlichen Haftstellen. - Das $\mathrm{Sm}^{3+}$-Spektrum kann bei ausschließlicher „Eu-Erregung“" beim Ausheizen

12 M. S chön, Verh. dtsch. physik. Ges. (3) 18, 70 [1937]; N. Ri e hl u. M. S c hön, Z. Physik 114, 685 [1939].

13 W. Schottky, Zusammenfassende Bearbeitung der bisherigen experimentellen Ergebnisse zu einer Theorie der Phosphore I. Pretzfeld 1944.

14 P. B r a u e r, Tagungsvortrag Physik. Ges. Stuttgart, 5./6. Juni; Physik. Bl. 4, 259 [1948]. 
nicht auftreten, weil dazu Defektelektronen thermisch von erregten Eu-Störtermen in das besetzte Band befördert werden müßten, was in Anbetracht der großen Energiestufe von etwa $2 \mathrm{eV}$ nicht geschieht. Wohl aber ist dies im Falle des SrS-Ce-Sm, SrS-Mn-Sm und SrS-Pr-Sm möglich (Pf. 9, Abb. 2); denn dort muß nach dem Erregungsspektrum der Aktivatorterm des Hauptaktivators Ce, Mn oder Pr wesentlich tiefer zu liegen kommen, so daß nach Entfernung eines Elektrons sein Abstand vom oberen Rand des besetzten Bandes offenbar kleiner ist als der
Abstand des mit einem Haftelektron besetzten SmStörterms vom unteren Rand des unbesetzten Bandes. Dann findet bei Erwärmung Rekombination an den Sm-Störstellen und Aussendung des $\mathrm{Sm}^{3+}$-Spektrums statt, während beim Ausleuchten die gleichen Vorgänge wie beim SrS-Eu-Sm ablaufen, also das Hauptaktivator-Spektrum ausgesandt wird. Die Kenntnis einiger Termabstände ist noch unsicher - insbesondere der Abstand beider Bänder —, so daß die Lage der Terme noch kleineren Änderungen unterworfen sein wird.

\title{
Theorie des Anklingens von Phosphoren, die für Ausleuchtung sensibilisiert sind
}

\author{
Von Peter Brauer \\ Aus der Osram G.m.b.H., Zweigstelle Mosbach (Baden) \\ (Z. Naturforschg. 5 a, 158-159 [1950]; eingegangen am 19. Dezember 1949)
}

\begin{abstract}
Es wird über Ergebnisse von Rechnungen berichtet, die den Ablauf des Ausleuchtvorgangs in für Ausleuchtung sensibilisierten Phosphoren verfolgen. Es ergibt sich u. a., daß bei Einsetzen der Ultrarotbestrahlung die Helligkeit des emittierten Lichtes auf einen Teil der Endhelligkeit springt, um dann den stationären bzw. Endwert allmählich zu erreichen. Die theoretischen Ergebnisse werden mit experimentellen Ergebnissen von $\mathrm{S} \cot t, \mathrm{Th}$ omp s on und E 1 li ck s on 2 verglichen.
\end{abstract}

$\mathrm{D}$ as Vorhandensein von Haftstellen, insbesondere mehrerer Haftstellenarten, wie sie in dem früher beschriebenen Modell der für Ausleuchtung sensibilisierten Phosphore ${ }^{1}$ enthalten sind, ist die Ursache dafür, daß das Aufleuchten des erregten Phosphors bei der einsetzenden Ultrarotbestrahlung träge erfolgt, worauf wir schon in unseren ersten Mitteilungen hingewiesen haben (Literatur s. in der unter ${ }^{1}$ zit. Arbeit). F. R. S cott, R. H. Thompson und R.T.Ellickso ${ }^{2}$ haben neuerdings die Zeitabhängigkeit gemessen und der Verf. sieht eine Stütze für das beschriebene Modell ${ }^{1}$ in der Tatsache, daß es ihm gelang, die teilweise ziemlich ungewöhnlich geformten, gemessenen Kurven aus dem Modell zu berechnen. Bei der Rechnung wurde vom erregten, aber nicht mehr nachleuchtenden Phosphor ausgegangen. Die gespeicherten Elektronen sitzen dann in tiefen (Sensibilisator-) Haftstellen. Das ausleuchtende Ultrarot hebt sie in ein höheres Niveau, von wo aus sie thermisch ins obere Band befördert werden. Von dort aus verteilen sie sich auf Leuchtstellen und alle Haftstellen. Letz-

1 P. B r a u e r, Z. Naturforschg. 5a, 156 [1950].

2 F. R. Scott, R. H. Thompson u. R. T. Ellickso n, J. opt. Soc. America 39, 64 [1949]. tere spalten ihrerseits wieder Elektronen ins Band ab. Die tieferen von ihnen füllen sich teilweise auf. Die Größenordnungen der Konstanten (Haftstellenkonzentrationen, Anlagerungswahrscheinlichkeiten, Term-

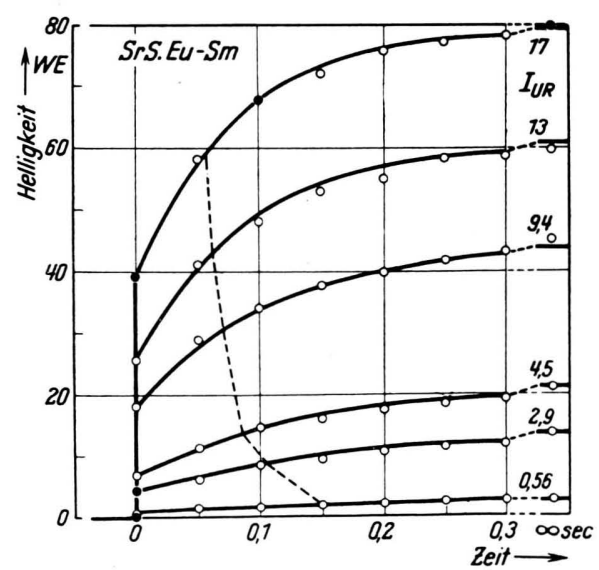

Abb. 1. Anfang des zeitlichen Verlaufs der Helligkeit der Lichtemission von erregtem SrS-Eu-Sm unter bei $t=0$ einsetzender konstanter Ultrarotbestrahlung der Intensität $I_{\mathrm{LR}}$. Theoretische Kurven. Eingetragene Meßpunkte nach Scott, Thompson und Ellickson2. Die Konstanten in den theoretischen Formeln wurden aus den fünf ausgefüllten Punkten bestimmt. 